\title{
Biodisponibilidad de lisina en dos pastas de soya con diferente nivel de actividad ureásica en pollos de engorda
}

\section{Lysine bioavailability in two soybean meals with different level of urease activity in broiler chicks}

\author{
Arturo Cortes Cuevasa, Carlos Martínez Amezcuab, Gabriela Gómez Verduzcoc, Carlos \\ López Coelloc, Ernesto Avila Gonzáleza
}

\begin{abstract}
RESUMEN
Para conocer la biodisponibilidad de lisina para pollos de dos pastas de soya (PSA y PSB), con diferente actividad ureásica (AU 0.11 y 0.17 unidades de incremento de pH), se realizó el presente experimento. Se utilizaron 210 pollitos Ross de 1 a 21 días de edad, los cuales se distribuyeron al azar en 7 tratamientos con tres repeticiones. Los tratamientos fueron: 1) dieta basal sorgo-soya-ajonjolí (deficiente en lisina), 2) dieta basal + 0.05\% de L-lisina, 3) dieta basal $+0.10 \%$ de L-lisina, 4) dieta basal+0.05\% de lisina a partir de PSA, 5) dieta basal+0.10\% de lisina a partir de PSA, 6) dieta basal+0.05\% de lisina a partir de PSB y 7) dieta basal+0.10\% de lisina a partir de PSB. Los resultados de crecimiento, se explicaron mediante la ecuación de regresión lineal múltiple, con los datos de ganancia de peso, consumos de lisina sintética o consumos de lisina a partir de las pastas de soya fue $Y=375.419+0.0378$ X1 + 0.0366 X2 + 0.0376 X3; en donde X1 correspondió a la complementación con L-lisina, X2 con la PSA (con adición de cascarilla cruda) y X3 con la PSB (con adición de cascarilla cocida). Al comparar la pendiente de la pasta de soya A o B, con la obtenida con L-lisina $(100 \%)$, se tuvieron biodisponibilidades de lisina de 97 y $99 \%$ para las pastas de soya A y B respectivamente. Estos resultados indican que la AU de la pasta de soya afecta la biodisponibilidad de lisina; a mayor actividad ureásica, menor es la disponibilidad.
\end{abstract}

PAlabras ClaVe: Pasta de soya, Actividad ureásica, Pollo de engorda, Biodisponbilidad de lisina, Parámetros productivos.

\section{ABSTRACT}

In order to study bioavailability of lysine of two soybeans meals (PSA and PSB) for broilers with different urease activity (UA of 0.11 and 0.17 units of pH change), an experiment was performed. Two hundred and ten (210) chicks Ross were used from 1 to $21 \mathrm{~d}$ of age, in a completely randomized design with 7 treatments with three replicates each one. Treatments were: 1) Basal diet sorghum-soybean meal (deficient in lysine), 2) Basal diet+0.05\% L-lysine, 3) Basal diet+0.10\% L-Iysine, 4) Basal diet+0.05\% lysine from PSA, 5) Basal diet+0.10\% lysine from PSA, 6) Basal diet+0.05\% lysine from PSB and 7) Basal diet+0.10\% lysine from PSB. The growth results obtained were explained by multiple linear regression equation, based on the data of weight gain, consumption of synthetic lysine and intakes of lysine from soybean meals: $Y=X 1+Y=375.419+0.0378$ X1 +0.0366 X2 + 0.0376 X3; where X1 accounted supplementation with L-lysine, X2 with PSA (with addition of raw hull) and X3 with PSB (with addition of cocked hull). Comparison of the PSA or PSB slopes with the L-lysine slope (i.e. $100 \%$ bioavailability) showed them all to have $>95 \%$ lysine availability: PS $A=97 \%$, PSB= $99 \%$. These results indicate that UA of soybean meal affected the bioavailability of lysine; therefore more than 0.12 urcase activity units of pH change, lower is the availability.

KEY WORDS: Soybean meal, Urease activity, Broilers, Lysine bioavailability, Performance.

Recibido el 5 de octubre de 2012. Aceptado el 17 de abril de 2012

a Centro de Enseñanza, Investigación y Extensión en Producción Avícola, Facultad de Medicina Veterinaria y Zootecnia, Universidad Nacional Autónoma de México. Salvador Díaz Mirón No. 89, Col. Zapotitlán, 13209. Tláhuac, DF. México. Tel: 58450029 - avilaernesto@yahoo.com. Correspondencia al último autor.

b Ajinomoto Biolatina Industria \& Comercio Ltda., México.

c Departamento de Producción Animal: Aves, Facultad de Medicina Veterinaria y Zootecnia, Universidad Nacional Autónoma de México. México. 


\section{INTRODUCCIÓN}

La pasta de soya es una excelente fuente de proteína para las aves, debido a que contiene un buen balance de aminoácidos esenciales $(A A)$, pero con la desventaja que contiene factores antinutricionales (inhibidores de tripsina y lectinas) que afectan el crecimiento de las aves si no es procesada adecuadamente(1). El procesamiento adecuado del frijol soya (tiempo de cocción y temperatura), inactiva los factores antinutricionales(2). Temperaturas excesivas destruyen AA sensibles al calor (metionina y lisina) y reducen el valor nutricional y la biodisponibilidad de $A A$, entre ellos la lisina, a causa de la reacción de Maillard(3-5). Se utilizan indicadores para control de calidad en el procesamiento de la pasta de soya (PS) como la actividad ureásica (AU); este índice es probablemente la prueba in vitro más utilizada para detectar el subprocesamiento de la PS. El índice de $\mathrm{AU}$ en pastas de soya evaluadas en el laboratorio con un rango de 0.05 a 0.20 de incremento en el $\mathrm{pH}$, indica un adecuado proceso, datos por encima de dichos valores indican subprocesamiento y datos por debajo un sobrecalentamiento(4).

Otros estudios varían en sus recomendaciones en cuanto a la relación AU con la calidad del procesamiento, los cuales reportan rangos de AU desde 0.03 hasta 0.30 unidades, indicando que las pastas de soya tuvieron un procesamiento adecuado de temperatura $(1,6,7)$. Sin embargo, algunos investigadores, señalan que el empleo de pasta de soya con una AU elevada (mayor a 0.20 unidades) en dietas para pollos, disminuye la digestibilidad de $A A$, entre ellos la lisina, y señalan que a mayor AU menor es la digestibilidad de este $A A$, disminuyéndose de 92 a $91 \%(4,5)$. Veltman et al(8), emplearon PS que contenían 0.06, 0.11 y 0.19 unidades de $A U$, encontrando un menor contenido de $A A$ esenciales y no esenciales con el valor más alto de AU. Por otra parte, Cortes et al(7), encontraron en PS producidas en México valores de $A U$ que oscilaban entre 0.11 a 0.22 , los cuales no afectaron el crecimiento de los pollos.

\section{INTRODUCTION}

Soybean meal's good amino acids (AA) balance makes it an excellent protein source in poultry diets, although it does have the disadvantage of containing antinutritional factors such as trypsin inhibitors and lectins. These can negatively affect animal growth if the SBM is not adequately processed(1) using a proper cooking time and temperature to deactivate these factors(2). Excessive heat, however, can cause Maillard reactions, destroying thermolabile AA such as methionine and lysine, reducing nutritional value and the bioavailability of some AA (e.g. lysine)(35). To control quality during soybean meal (SBM) processing, indicators such as urease activity (UA) are used. Probably the most widely used in vitro test for SBM under-processing, UA is based on $\mathrm{pH}$, with a 0.05 to 0.20 unit increase in $\mathrm{pH}$ indicating adequate processing. Values above this range indicate under-processing and those below it over heating(4).

Wider UA value ranges (0.03 to 0.30 ) have been reported as indicating an adequate processing temperature $(1,6,7)$. Nonetheless, use of SBM with a UA value higher than 0.20 units in diets for chickens is reported to decrease $A A$ digestibility (including lysine), with higher UA values lowering AA digestibility by 91 to $92 \%(4,5)$. This coincides with a study of UA in chicken diets containing SBM with $0.06,0.11$ or 0.19 UA units, which found lower essential and nonessential AA levels at higher UA values(8). However, in another study of SBM in chicken diets, UA values between 0.11 and 0.22 had no effect on growth(7).

Amino acid bioavailability is measured through growth tests and the slope technique. A single synthetic $A A$ is added to a control diet deficient in this AA. The tested ingredient is then added to the control diet at one or various levels, the resulting growth response documented and compared to the response in treatments using only the crystalline $A A^{(9-11)}$.

The present study objective was to evaluate lysine bioavailability in SBM containing raw or 
Para medir la biodisponibilidad de un aminoácido, se emplean pruebas de crecimiento y la técnica de la pendiente. Un solo aminoácido sintético, se suplementa a varios niveles a una dieta basal deficiente en ese aminoácido, que sirve como curva estándar. El ingrediente a probar se adiciona en uno o más niveles a la dieta basal como fuente del aminoácido a probar, y el crecimiento de los pollitos que recibe del ingrediente a probar, se compara con el obtenido de los pollitos alimentados con el aminoácido cristalino(9-11).

Con estos antecedentes, se planteó el presente estudio con la finalidad de evaluar la biodisponibilidad de lisina de una pasta de soya, a la que se le adicionó el mismo nivel de cascarilla de soya, cruda o procesada térmicamente, en dietas para pollos de engorda en crecimiento, empleando el método de comparación de pendientes con regresión lineal múltiple.

\section{MATERIALES Y MÉTODOS}

La investigación se realizó en el Centro de Enseñanza, Investigación y Extensión en Producción Avícola (C.E.I.E.P.Av.) de la Facultad de Medicina Veterinaria y Zootecnia de la Universidad Nacional Autónoma de México, localizado a 2,250 msnm. entre los paralelos $19^{\circ} 15^{\prime}$ latitud Oeste; clima templado húmedo $\mathrm{CW}$, siendo enero el mes más frío y mayo el más caluroso; su temperatura promedio anual es de $16{ }^{\circ} \mathrm{C}$ y con una precipitación pluvial anual media de $747 \mathrm{~mm}$.

Se utilizaron 210 pollitos mixtos de 1 a 21 días de edad, de la estirpe Ross obtenidos de una incubadora comercial. Las aves fueron distribuidas en 21 lotes de 10 pollos cada una (mitad machos y mitad hembras), alojados en pisos de jaulas en baterías Petersime con temperatura controlada por termostato. Los pollos se distribuyeron completamente al azar, en 7 tratamientos cada uno con tres repeticiones de 10 aves cada una: thermally processed soybean hulls in diets for broiler chickens using slope comparison with a multiple linear regression.

\section{MATERIALS AND METHODS}

The experiment was done at the Center for Poultry Production Teaching, Research and Extension (Centro de Enseñanza, Investigación y Extensión en Producción Avícola - (CEIEPAv) of the Faculty of Veterinary Medicine of the National Autonomous University of Mexico (Universidad Nacional Autónoma de México UNAM). The installations are located at 2,250 $\mathrm{m}$ asl in the Valley of Mexico $\left(28^{\circ} 13^{\prime} \mathrm{N}\right.$; $98^{\circ} 36^{\prime}$ W). Climate is humid temperate ( $\mathrm{CW}$ ) with the lowest temperatures in J anuary and the highest in May. Average annual temperature is $16{ }^{\circ} \mathrm{C}$ and average annual rainfall is $747 \mathrm{~mm}$.

Experimental animals were a mixed batch of Ross strain broiler chicks ( $n=210 ; 1$ to $21 \mathrm{~d}$ of age) obtained from a commercial incubator, and housed in Petersime Batteries cage floors with thermostatically controlled temperature. They were distributed in 21 pens with ten animals each, half of each pen being male and the other half female. Pens were distributed randomly into seven treatments with three replicates each and ten chicks per replicate. 1) Control base diet; 2) Control $+0.05 \%$ L-lysine; 3) Control $+0.10 \%$ L-lysine; 4) Control $+0.05 \%$ lysine from SBM A (+ raw hulls); 5) Control + $0.10 \%$ lysine from SBM A (+ raw hulls); 6) Control $+0.05 \%$ lysine from SBM B (+ processed hulls); and 7) Control $+0.10 \%$ lysine from SBM B (+processed hulls).

A lysine-deficient control base diet containing sorghum, SBM and sesame paste was used as the control and all experimental diets were formulated using this diet (Table 1). Protein, amino acids(12), lysine and $U A^{(13)}$ analyses were done of all ingredients before base and experimental diet formulation (Table 2). The experimental diets were formulated by supplementing the base diet with crystal L-lysine $(\mathrm{HCl})$, SBM A (containing raw hulls) or SBM B 
1) dieta basal; 2) como $1+0.05 \%$ de L-lisina; 3) como $1+0.10 \%$ de L-lisina; 4) como $1+$ $0.05 \%$ de lisina a partir de la pasta de soya $A$ (+cascarilla cruda); 5) como $1+0.10 \%$ de lisina a partir de las pasta de soya A (+cascarilla cruda); 6) como $1+0.05 \%$ de lisina a partir de las pasta de soya B (+cascarilla procesada); 7) como $1+0.10 \%$ de lisina a partir de las pasta de soya $\mathrm{B}$ (+cascarilla procesada).

Previo a la formulación de la dieta basal, se realizaron análisis de proteína y aminoácidos de los ingredientes(12). Los análisis de proteína, lisina y $A U(13)$ en las PS aparecen en el Cuadro 2. El contenido de lisina de la PSA y PSB, se empleó para el cálculo de la cantidad a utilizar de estas harinas, para cubrir el porcentaje de lisina evaluado.

Para obtener las dietas experimentales, a una dieta basal sorgo+pasta de soya+ pasta de ajonjolí solamente deficiente en lisina (Cuadro 1), se le hicieron las suplementaciones de L-lisina $(\mathrm{HCl})$ o lisina a partir de las pastas de soya $A$ o B como fuente de este aminoácido. También se muestra como se hicieron las inclusiones de lisina cristalina y lisina a partir de las pastas de soya A y B a expensas del azúcar.

En el Cuadro 2, se muestra la composición de las dos pastas de soya A y B con $44 \%$ de proteína, las cuales fueron elaboradas a partir de una pasta de soya con $48.5 \%$ de proteína, $2.98 \%$ de lisina y AU de 0.12; se utilizó $87.3 \%$ de inclusión, a la que se le adicionó una cantidad fija de una cascarilla de soya, misma que se manejó cruda ( $12.7 \%$ de inclusión) con $10.76 \%$ de proteína, $0.68 \%$ de lisina y 0.5 de AU para elaborar la pasta de soya A, o bien, la misma cascarilla de soya pero cocida ( $12.7 \%$ de inclusión) en autoclave con la misma cantidad de nutrientes, pero con 0.12 de AU para elaborar la pasta de soya $B$.

Se llevaron registros de ganancia de peso, consumo de alimento y conversión alimenticia. Los datos de las variables obtenidas al final del estudio, fueron inicialmente analizados (containing thermally-processed hulls) in replacement of sugar. The calculations for SBM $A$ and SBM $B$ inclusion levels were made based on their lysine contents. Using the same source SBM (48.85 \% protein, $2.98 \%$ lysine, 0.12 UA), both the SBM $A$ and SBM $B$ were

Cuadro 1. Composición de la dieta basal (deficiente en lisina) para pollos en crecimiento

Table 1. Composition of lysine-deficient base diet for growing broiler chicks

\begin{tabular}{|c|c|}
\hline Ingredients & $\mathrm{kg} / \mathrm{t}$ \\
\hline Sorghum & 458.555 \\
\hline Soybean meal & 92.982 \\
\hline Sesame paste & 331.434 \\
\hline Vegetable oil & 48.803 \\
\hline Orthophosphate & 18.626 \\
\hline Calcium carbonate & 11.259 \\
\hline Salt & 4.114 \\
\hline Choline chloride $60 \%$ & 1.000 \\
\hline Vitamins ${ }^{*}$ & 1.000 \\
\hline Minerals ${ }^{\star \star}$ & 0.500 \\
\hline DL-methionine & 0.288 \\
\hline L-Threonine & 0.560 \\
\hline Sugar ${ }^{\star \star \star}$ & 30.480 \\
\hline Bacitracin & 0.288 \\
\hline Antioxidant & 0.100 \\
\hline Total & 1000 \\
\hline Nutrients: & Analysis \\
\hline Metabolizable energy, kcal/kg & 3000 \\
\hline Crude protein, \% & 22.00 \\
\hline Methionine, \% & 0.479 \\
\hline Met+Cys, \% & 0.900 \\
\hline Lysine, \% & 0.700 \\
\hline Calcium, \% & 1.000 \\
\hline Available phosphorous, \% & 0.500 \\
\hline Sodium, \% & 0.180 \\
\hline
\end{tabular}

*Amounts / kg: vitamin A, 3000000 IU; vitamin $D_{3}, 750000$ IU; vitamin $\mathrm{E}, 6000 \mathrm{IU}$; vitamin $\mathrm{K}_{3}, 1.0 \mathrm{~g}$; riboflavin, $4 \mathrm{~g}$; $\mathrm{B}_{12}, 0.060 \mathrm{~g}$; pyridoxine, $3.0 \mathrm{~g}$; calcium pantothenate, $13.0 \mathrm{~g}$; niacin, $25 \mathrm{~g}$; biotin, $0.063 \mathrm{~g}$; choline chloride, $250 \mathrm{~g}$.

** Amounts / kg.: selenium, $0.2 \mathrm{~g}$; cobalt, $0.1 \mathrm{~g}$; iodine, $0.3 \mathrm{~g}$; copper, $10 \mathrm{~g}$; zinc, $50 \mathrm{~g}$; iron, $100 \mathrm{~g}$; manganese, $100 \mathrm{~g}$; vehicle sufficient for $1000 \mathrm{~g}$.

*** Sugar was replaced to supplement with L-lysine HCL (78.8\%) for T1 (0.0\%), T2 (0.05\%) and T3 (0.10\%), SBM A for T4 (1.861\%) and T5 (3.723\%), or SBM B for T6 (1.861\%) and T7 (3.723\%). 
empleando el procedimiento de análisis de varianza para diseños completamente al azar utilizando el paquete estadístico de SPSS(14). Los datos de ganancia de peso y consumo de lisina, se sometieron a un análisis de regresión lineal múltiple. El consumo de lisina (mg/pollo) se calculó a partir de la inclusión de lisina sintética y de las pastas de soya A y B. La biodisponibilidad de lisina se estimó por la metodología de comparación de pendientes con regresión lineal múltiple(9), utilizando los datos de ganancia de peso de los pollitos ( $Y=$ variable dependiente), $\beta_{0}=$ como la ordenada de origen con el consumo de lisina sintética ( $\beta_{1} X_{1}=$ curva estándar) y la comparación con los del consumo de lisina (variable independiente) de las pastas de soya $A$ o $B\left(\beta_{2} X_{2}+\beta_{3} X_{3}\right)$ respectivamente.

\section{RESULTADOS}

Los datos promedios por ave en 21 días de experimentación para ganancia de peso, consumo de alimento, consumo de lisina y eficiencia alimenticia aparecen en el Cuadro 3. Se puede apreciar, que los aumentos de peso y eficiencias alimenticias mejoraron con la suplementación de lisina sintética o con la suplementación de las pastas de soya como fuente de lisina de manera equivalente. La ganancia de peso en relación al consumo de lisina sintética suplementada y el consumo de lisina con las pastas de soya se explicó $(\mathrm{P}<0.049)$ por medio de la ecuación regresión $Y=375.419+0.0378 X 1+0.0366 X 2+0.0376$ $X 3$; en donde $X 1$ correspondió a la complementación con L-Lisina, X2 con la PSA (con adición de cascarilla cruda) y X3 con PSB (con adición de cascarilla cocida). La Figura 1, muestra que al comparar la pendiente de la PSA o PSB, con la pendiente obtenida con Llisina (100\%), se obtuvieron digestibilidades de lisina de 97 y $99 \%$ para PSA y PSB respectivamente; estos resultados indican que el subprocesamiento de la pasta de soya afecta la biodisponibilidad de este aminoácido, es decir a mayor AU menor es la digestibilidad. formulated with $87.3 \%$ SBM and $12.7 \%$ hulls. Protein content was $44 \%$, and lysine content $2.98 \%$ in the SBM A and SBM B, but UA was determined by hull condition. The SBM A was formulated using raw hulls (10.76\% protein, $0.68 \%$ lysine, $0.5 \mathrm{UA})$, resulting in a UA of 0.17 . The SBM B was formulated using cooked hulls (10.76\% protein, $0.68 \%$ lysine, 0.12 $\mathrm{UA}$ ), resulting in a UA of 0.12 (Table 2).

Weight gain, feed intake and feed conversion data were collected for the experimental animals and initially analyzed using an analysis of variance (ANOVA) for completely random data (SPSS statistics package)(14). Lysine intake (mg/ chick) was calculated based on inclusion levels for the synthetic lysine and SBM A and SBM B. Weight gain and lysine intake data were analyzed with a multiple linear regression. Lysine bioavailability was estimated by comparing slopes to the multiple linear regression(9), using chick weight gain data ( $Y=$ dependent variable), the source ordinate with synthetic lysine intake $\left(\beta_{1} X_{1}=\right.$ standard curve), and a comparison with lysine intake (independent variable) in SBM A or SBM B.

\section{RESULTS}

After the 21-d feeding trial, weight gain and feed efficiency improved to an equivalent degree

Cuadro 2. Contenido de proteína, lisina y actividad ureásica en las pastas de soya con la adición de cascarilla cruda (A) 0 cocida (B)

Table 2. Protein and lysine content, and urease activity in soybean meal (SBM) containing added raw hulls $(A)$ or cooked hulls (B)

\begin{tabular}{lccc}
\hline SBM & Protein (\%) & Lysine (\%) & Urease activity \\
\hline A & 44 & 2.98 & 0.17 \\
B & 44 & 2.98 & 0.12 \\
\hline
\end{tabular}

SBM A was formulated using a SBM (87.3\%) containing $48.85 \%$ protein, $2.98 \%$ lysine and 0.12 UA, and adding raw soybean hulls $(12.7 \%)$ containing $10.76 \%$ protein, $0.68 \%$ lysine and $0.5 \mathrm{UA}$.

SBM B was formulated using a SBM (87.3\%) containing $48.85 \%$ protein, $2.98 \%$ lysine and $0.12 \mathrm{UA}$, and adding cooked soybean hulls (12.7\%) containing $10.76 \%$ protein, $0.68 \%$ lysine and $0.12 \mathrm{UA}$. 
Cuadro 3. Datos promedio de 0 a 21 días de edad en pollos de engorda alimentados con diferentes niveles de lisina Table 3. Average growth performance data for broiler chicks fed diets containing different lysine levels for 21 days

\begin{tabular}{lcccc}
\hline Treatment & Final weight $(\mathrm{g})$ & Weight gain $(\mathrm{g})$ & Lysine intake $(\mathrm{mg})$ & Feed efficiency \\
\hline 1. Base diet (Control) & $671 \pm 13.4$ & $631 \pm 12.6$ & $7010 \pm 280$ & $0.72 \pm 0.014$ \\
2. C+0.05\% L-lysine & $695 \pm 13.9$ & $655 \pm 13.1$ & $7380 \pm 273$ & $0.75 \pm 0.015$ \\
3. C+0.10\% L-lysine & $720 \pm 13.7$ & $680 \pm 12.9$ & $8000 \pm 229$ & $0.77 \pm 0.015$ \\
4. C+0.05\% lysine SBMA & $691 \pm 14.5$ & $651 \pm 12.7$ & $7240 \pm 286$ & $0.76 \pm 0.014$ \\
5. C+0.10\% lysine SBMA & $720 \pm 14.0$ & $680 \pm 13.6$ & $8010 \pm 316$ & $0.76 \pm 0.015$ \\
6. C+0.05\% lysine SBMB & $697 \pm 13.9$ & $657 \pm 12.8$ & $7660 \pm 295$ & $0.73 \pm 0.015$ \\
7. C+0.10\% lysine SBMB & $715 \pm 13.6$ & $675 \pm 13.5$ & $8012 \pm 312$ & $0.76 \pm 0.014$ \\
\hline
\end{tabular}

Multiple linear regression for weight gain $(\mathrm{Y} ; \mathrm{g})$ and supplementation with synthetic lysine $\left(\mathrm{X}_{1}=\mathrm{T} 1, \mathrm{~T} 2, \mathrm{~T} 3\right)$ or $\mathrm{SBM} A$ and $\mathrm{B}$ $\left(\mathrm{X}_{2}=\mathrm{T} 1, \mathrm{~T} 4, \mathrm{~T} 5 ; \mathrm{X}_{3}=\mathrm{T} 1, \mathrm{~T} 5, \mathrm{~T} 6\right)$ :

$Y=375.419+0.0378 x_{1}+0.0366 x_{2}+0.0376 x_{3} R^{2}=0.653(P<0.049) ;$ where: $X_{1}=100 \%$ L-lysine; $X_{2}=97 \%$ SBM A; $X_{3}=99 \%$ SBM B.

\section{DISCUSIÓN}

Condiciones apropiadas de procesamiento del frijol soya (tiempo de cocción y temperatura), inactivan los factores antinutricionales, lo que resulta en un incremento en el crecimiento de los pollos $(15,16)$. Veltman et al( $(8)$, indican que el bioensayo en pollos es el mejor método para evaluar el valor nutritivo de pastas de soya que pruebas in vitro. Un alto contenido de los inhibidores de proteasas, especialmente inhibidores de la tripsina afectan adversamente la digestibilidad de la proteína y $\mathrm{AA}^{(3)}$. La gran mayoría de las investigaciones, mencionan que cuando la pasta de soya recibe un adecuado tratamiento térmico, disminuye la $\mathrm{AU}$ y se sugieren valores de 0.05-0.20 unidades de incremento de $\mathrm{pH}$, para no disminuir la biodisponibilidad de aminoácidos, entre ellos lisina y metionina como AA indispensables; además, para no afectar el rendimiento productivo(3-5).

La ureasa es una enzima en las pastas de soya, que puede causar toxicidad por amoniaco en rumiantes alimentados con dietas conteniendo urea. Aunque no tiene ninguna función fisiológica en animales monogástricos, es usualmente deseable reducir la actividad ureásica a niveles muy bajos en las PS empleadas en la alimentación de las aves. Las in the L-lysine, SBM A and SBM B treatments (Table 3). Weight gain in relation to lysine intake was explained $(P<0.049)$ by the regression equation $Y=375.419+0.0378 X 1+0.0366$ $X 2+0.0376 X_{3}$; where $X 1$ corresponds to L-lysine supplementation, X2 to SBM A (i.e. raw hulls), and X3 to SBM B (i.e. processed hulls). Compared to the L-lysine slope (100 \%), the SBM A slope indicated $97 \%$ lysine digestibility and the SBM B slope $99 \%$. Clearly, under processing SBM affects lysine digestibility since values are lower as UA increases.

Figura 1. Porcentaje de biodisponibilidad de Lisina de dos pastas de soya respecto a la L-lisina $\mathrm{HCL}$

Figure 1. Lysine disponibility rate from two soybeans meals

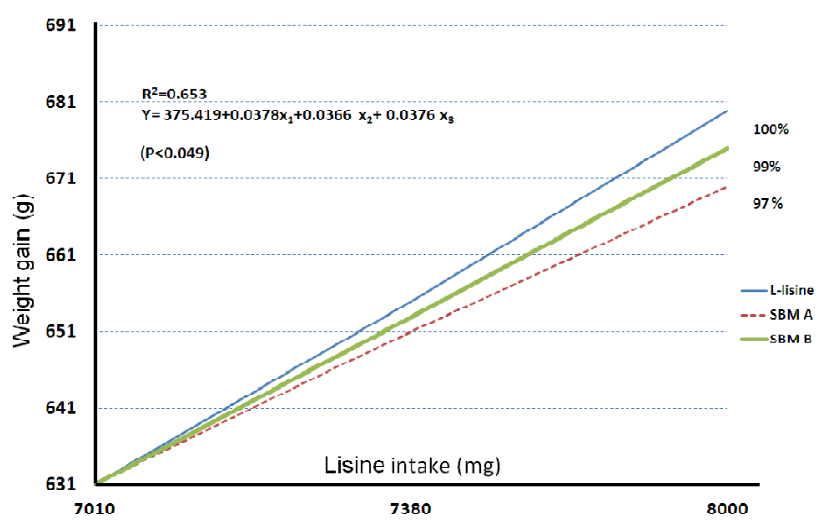


plantas procesadoras de frijol soya en Estados Unidos consideran que una AU de 0.10 es satisfactoria y segura para incluirla en dietas para aves. Fasina et al (1), señalan que valores de $A U$ entre 0.031 a 0.088 es indicativo de un adecuado proceso en las PS y que éstas contienen niveles muy bajos de lectinas.

Los datos en el presente estudio, muestran que se afectó la biodisponibilidad de lisina de las pastas de soya A y B (97 y $99 \%$ respecto a L-lisina como $100 \%$ ) cuando los pollos consumieron dietas con pasta de soya que contenía ( 0.17 y 0.12 unidades de AU). Lo anterior concuerda con lo obtenido por Ruiz et al(17), quienes encontraron que 0.10 unidades de $A U$, son adecuadas para generar una óptima calidad en pastas de soya y soyas integrales, lo que se refleja en un mejor crecimiento en los pollos de engorda y digestibilidad de aminoácidos.

Con base a los resultados obtenidos en la presente investigación, se sugiere que el contenido de AU en la pasta de soya deberá ser igual o menor a 0.12 unidades de incremento de $\mathrm{pH}$, para una mayor biodisponibilidad de lisina.

\section{CONCLUSIONES E IMPLICACIONES}

Los datos indican que el bioensayo en pollos en crecimiento de relación de pendientes a una curva estándar de lisina sintética, permite detectar diferencias en la calidad nutritiva de la pasta de soya con diferentes niveles de actividad ureásica; la pasta de soya con el nivel más alto de $A U(0.17)$ tuvo una menor disponibilidad de lisina, lo que impactó en el crecimiento y la eficiencia alimenticia de pollos en crecimiento.

\section{LITERATURA CITADA}

1. Fasina YO, Classen HL, Garlich JD, Swaisgood HE, Clare DA. Investigating the possibility of monitoring lectin levels in commercial soybean meals intented for poultry feeding using steam-heated soybean meal as a model. Poult Sci 2003; 82:648-656.

\section{DISCUSSION}

When soybean is processed under appropriate conditions (temperature and time) its antinutritional factors are inactivated, allowing improved growth performance in chickens $(15,16)$. Bioassays are the best in vitro method for evaluating SBM nutritional value(8). High protease inhibitor content, particularly trypsin inhibitors, negatively affects protein and AA digestibility(3). Urease activity decreases when SBM receives proper thermal treatment. Values from 0.05 to 0.20 units increase in $\mathrm{pH}$ are recommended so as not to lower AA bioavailability, especially the essential AA lysine and methionine, and not to affect production yields(3-5).

Urease is an enzyme found in SBM that can cause ammonia poisoning in ruminants fed diets containing urea. Although it has no physiological function in monogastric animals, it is usually best to maintain very low UA levels in SBM used in poultry feed. Soybean processing plants in the United States use a 0.10 UA as satisfactory and safe for poultry diets. Values for UA between 0.031 and 0.088 are indicative of adequate soybean processing and that lectin levels are sufficiently low(1).

Lysine bioavailability in SBM A (0.17 UA) and SBM B (0.12 UA) treatments was lower than in the L-lysine treatment. This coincides with a study indicating that a $0.10 \mathrm{UA}$ is adequate for optimum quality soybean and whole SBM, as shown in improved growth performance in broilers and AA digestibility(17).

Based on the present results, UA levels in SBM must be equal to or less than 0.12 units increase in $\mathrm{pH}$ to ensure greater lysine bioavailability.

\section{CONCLUSIONS AND IMPLICATIONS}

The bioassay data generated here using broiler chicks and comparison of slopes to a synthetic lysine standard curve identified differences in nutritional quality for SBM with different urease 
2. Cuca GM, Avila GE, Pro MA. Alimentación de las aves. 2da ed. Universidad Autónoma Chapingo, Chapingo, Estado de México. 2009.

3. Coca-Sinova A, Jiménez -Moreno $E$, González -Alvarado J M, Frikha M, Lázaro R, Mateos GG. Influence of source of soybean meal and lysine content of the diet on performance and total tract apparent retention of nutrients in broilers from 1to 36 days of age. Poult Sci 2010;89:1440-1450.

4. Heuisuck L, Garlich JD. Effect of overcooked soybean meal on chicken performance and amino acid availability. Poult Sci 1992; 71: 499-508.

5. Anderson $\mathrm{HJ}$, Zhang $\mathrm{Y}$, Parsons $\mathrm{CM}$. Effect of heating on nutritional quality of conventional and Kunitz tyripsin inhibitor-free soybeans. Poutl Sci 1992;71:1700-1709.

6. Batal $A B$, Douglas MW, Engram AE, Parsons $C M$. Protein dispersibility index as an indicator of adequately processed soybean meal. Poult Sci 2000;79:1592-1596.

7. Cortes CA, Celis GA, Avila GE, Morales BE. Valor nutrimental de cuatro pastas de soya procesadas en diferentes estados de la República Méxicana. Vet Mex 2002;33:209-217.

8. Veltman JR, Blaine $\mathrm{CH}$, Tanksley TD, Knabe D, Linton SS. Comparison of the nutritive value of different heat-treated comercial soybean meals: Utilization by chicks in practical type rations. Poult Sci 1986;65:1561-1570.

9. Cortes CA, Martínez AC, Avila GE. Biodisponibilidad de lisina para el pollo en crecimiento de cuatro harinas de subproductos avícolas. Rev Mex Cienc Pecu 2012;2: 259-266.

10. Coca-Sinova A, Valencia DG, Jiménez -Moreno $E$, Lázaro $R$, Mateos GG. Apparent ileal digestibility of energy, nitrogen, and amino acids of soybean meals of different origin in broilers. Poult Sci 2008;87:2613-2623.

11. Parsons MC. Methods for determining amino acid bioavailability in poultry with emphasis on the ileal amino activity. The SBM with the higher (0.17) urease activity had lower lysine availability which negatively impacted growth and feed efficiency in growing chickens.

End of english version

acid digestibility technique. Dep Anim Sci. University of Illinois. Primer seminario de actualización en el uso de aminoácidos cristalinos. Querétaro, Qro. 2007:1-12.

12. Degussa. Nutritional reports and animal nutrition. Teterboro NJ, US: Degussa Co; 1986.

13. American Oil Chemists Society. Urease activity. Official Method Ba 9-58. American Oil Chemists Society, Champaign, IL, 1980.

14. SPSS Inc. SPSS for Windows (computer program) versión 8.0. spssinc 1989-1997.

15. Parsons CM, Hashimoto K, Wedekind KJ, Han Y, Baker DH. Effect of overprocessing on availability of amino acids and energy in soybean meal. Poult Sci 1992; 71:133-140.

16. Aburto A, Vazquez M, Dale NM. Strategies for utilizing overprocessed soybean meal: II. Lysine supplementation. J Appl Poult Res 1998; 7:196-201.

17. Ruiz N, Belalcazar F, Díaz GJ. Quality control parameters for commercial full-fat soybeans processed by two different methods and fed to broilers. J Appl Poult Res 2004; 13:443450. 